\title{
PERSISTÊNCIA DE MAMÍFEROS DE MÉDIO E GRANDE PORTE EM FRAGMENTOS DE FLORESTA OMBRÓFILA MISTA NO ESTADO DO PARANÁ, BRASIL ${ }^{1}$
}

\author{
Carlos Rodrigo Brocardo² e José Flávio Cândido Júnior ${ }^{3}$
}

\begin{abstract}
RESUMO - A Floresta Ombrófila Mista hoje é representada por fragmentos remanescentes do que no passado foi uma área contínua de cerca de $200.000 \mathrm{~km}^{2}$. Espécies de mamíferos de médio e grande porte que habitam essa floresta têm sofrido com os impactos decorrentes do processo de perturbação e degradação ambiental. Nesse contexto, este estudo realizou esforços para verificar quais espécies de mamíferos continuam habitando os remanescentes dessa formação vegetal na região Oeste do Estado do Paraná. Para tanto, foram amostrados três fragmentos florestais, com 520, 405 e 135 ha, compostos por trechos de floresta primária mesclados com floresta secundária. Os registros dos mamíferos foram feitos de maneiras direta (visualizações) e indireta (vestígios, análise de pelos e entrevistas com moradores locais), resultando na confirmação da ocorrência de 32 espécies (incluindo uma exótica e duas de pequeno porte), inseridas em oito ordens e 18 famílias. Nove dessas espécies estão sob o risco de extinção no Paraná e cinco configuram com dados insuficientes na Lista de Espécies Ameaçadas do Estado. Os resultados do estudo indicaram grande importância dos fragmentos na conservação de diversas espécies de mamíferos de médio e grande porte na Floresta Ombrófila Mista paranaense, mas, devido às pressões que essa área sofre, há a necessidade de ações públicas concretas e imediatas para garantir a persistência das populações de mamíferos.
\end{abstract}

Palavras-chave: Fragmentação florestal, Araucaria angustifolia e Caça.

\section{PERSISTENCE OF LARGE AND MEDIUM MAMMALS IN ARAUCARIA FOREST FRAGMENTS IN PARANÁ STATE, BRAZIL}

\begin{abstract}
The Araucaria Forest is now represented by remnants of what was a continuous area of about $200,000 \mathrm{~km}^{2}$. Medium and large mammals inhabiting this forest have suffered the impacts from the disturbance process and environmental degradation. Thus, this study determined which medium and large mammal species still inhabit the remnants of this vegetation type in Western Paraná State. Three study areas, with 520, 405 and 135 hectares, consisting of stretches of primary forest mixed with secondary forest, were considered for mammal inventory. The evidence of mammals was verified directly (views) and indirectly (traces, trichology and interviews with local dwellers). It was recorded 32 species (including one exotic and two small ones), belonging to eight orders and 18 families. Nine animals of these species are at risk of extinction in the Paraná State and five configure with insufficient data in the List of Endangered Species of the state. The results of this study indicate the great importance of these forest fragments in the conservation of several medium and large mammals in the Araucaria Forest of Paraná State. Nevertheless, due to the negative pressures that these areas have been suffering, immediate and concrete public actions are required to ensure the maintenance of these mammal populations.
\end{abstract}

Keywords: Forest fragmentation, Araucaria angustifolia and Hunting.

\footnotetext{
${ }^{1}$ Recebido em 07.03.2011 e aceito para publicação em 29.03.2012

${ }^{2}$ Mestre em Ciencias Biologicas (Zoologia), pela Universidade Estadual Paulista Júlio de Mesquita Filho, UNESP, Brasil. E-mail: <carlosbrocardo@hotmail.com>.

${ }^{3}$ Universidade Estadual do Oeste do Paraná, UNIOESTE, Brasil. E-mail: <jose.candido@unioeste.br>.
} 


\section{INTRODUÇÃO}

A fragmentação e perda de habitats naturais são as maiores ameaças à conservação de espécies selvagens (FAHRIG, 2003) e implicam perda de biodiversidade (TURNER, 1996). Vertebrados são particularmente afetados pela fragmentação, e os fragmentos impõem restrições à permanência de espécies devido à limitação da área de vida, efeitos de borda, escassez de recursos e restrição à dispersão provocada pela matriz circundante (CÂNDIDOJR., 2000; FAHRIG, 2003; COSTA et al., 2005).

Em situações de fragmentação dos ambientes naturais, mamíferos de médio e grande porte tendem a enfrentar um problema adicional, que é a forte pressão da caça ilegal, favorecida em áreas restritas como fragmentos (CHIARELLO, 1999; CULLEN-JR. et al., 2000; CULLEN-JR. et al., 2001; PERES, 2001; ROCHAMENDES et al., 2005). Assim, a sobrevivência dessas espécies, que são preferidas pelos caçadores, torna-se ainda mais ameaçada.

Mesmo ambientes naturais muito fragmentados e pequenos remanescentes podem, contudo, abrigar determinadas espécies e servir de "trampolins" para o deslocamento de indivíduos (CHIARELLO, 2000a). Dessa forma, conhecer quais espécies são encontradas em fragmentos relictuais de vegetação é fundamental para a implementação de planos de manejo e conservação (CHIARELLO, 2000a). Existe, entretanto, grande lacuna de conhecimento da fauna de determinadas regiões e formações fitogeográficas, com destaque para a Mata Atlântica (TABARELLI et al., 2005; GALETTI et al., 2009).

A Floresta Ombrófila Mista (FOM), também chamada de Floresta de Araucárias ou Mata dos Pinhais, é um bom exemplo dessa situação. Essa formação florestal que ocorria em maior expressão nos Estados do Sul do Brasil e em pontos relictuais do Sudeste brasileiro e pequena porção do Norte da Argentina sofreu intensa fragmentação durante os séculos XIX e XX, sobretudo pela extração do pinheiro-do-paraná (Araucaria angustifolia), árvore que caracteriza essa formação (KOCH; CORRÊA, 2002; MÄHLER-JR., LAROCCA, 2009). No Oeste do Paraná, a fragmentação da FOM teve início em meados de 1930, com a exploração madeireira. Esse ciclo teve seu ápice nas décadas de 1950 e 1960, e após a década de 1970 a atividade madeireira foi substituída pela agricultura (SPERANÇA, 1992; GREGORY et al., 2004).
Atualmente restam pouco mais que $12 \%$ de toda a área original coberta pela FOM, e apenas 3\% desses remanescentes estão em Unidades de Conservação (RIBEIRO et al., 2009), o que a torna uma das áreas prioritárias para conservação e esforços de pesquisa no bioma Mata Atlântica (COSTA et al., 2005; GALETTI et al., 2009; RIBEIRO et al., 2009).

Neste trabalho foram apontados os mamíferos de médio e grande de porte que persistem em três fragmentos de FOM no Oeste paranaense, bem como avaliadas as principais ameaças que incidem sobre tais espécies e sobre os fragmentos estudados.

\section{MATERIAL E MÉTODOS}

\section{1. Área de estudo}

O estudo foi desenvolvido em três fragmentos de FOM, no Município de Cascavel, região Oeste do Estado do Paraná. O clima na área de estudo é classificado como Cfa por Köppen, com precipitação média de 1.8002.000 mm anuais, sem estação seca definida e temperatura média anual variando de 19 a $21^{\circ} \mathrm{C}$, com a ocorrência de geadas no inverno. Na localidade, a FOM sofre influência da Floresta Estacional Semidecidual, formando um ecótono com a presença de espécies arbóreas como a peroba (Aspidosperma polyneuron) e o angico (Anadenanthera colubrina) (CASTELLA; BRITEZ, 2004). As atividades econômicas predominantes são a agricultura de cereais, a suinocultura, a avicultura e a pecuária de corte e leiteira.

Duas das áreas amostradas correspondem à Área de Proteção Permanente (APP) e Reserva Legal (RL) de uma propriedade particular. Essas áreas foram denominadas neste estudo Mata do Rio d’Oeste (MRO; $2^{\circ} 09^{\prime}$ S e $\left.53^{\circ} 15^{\prime} \mathrm{O}\right)$, com 520 ha; e Mata do Rio Tormenta (MRT; 2510'S e 53¹5’O), com 405 ha (Figura 1), com altitude local variando de 650 a 800 m. Nas duas áreas ocorrem trechos de florestas primária e secundária, porém sem o predomínio do pinheiro-do-paraná (Araucaria angustifolia) devido à exploração madeireira no passado. A terceira área é uma Unidade de Conservação (UC) Municipal de Cascavel, PR, o Centro de Educação Ambiental Suely Marcondes de Moura Festugato (CEA; $25^{\circ} 00^{\prime}$ 'S e $53^{\circ} 17^{\prime} \mathrm{O}$ ), com 135 ha e altitude em torno de 800 m (Figura 1). Essa UC foi criada em 1998, sendo anteriormente área particular, que sofreu exploração seletiva de madeira. Hoje, encontra-se em regeneração natural com grande quantidade de taquaras e epífitas. 


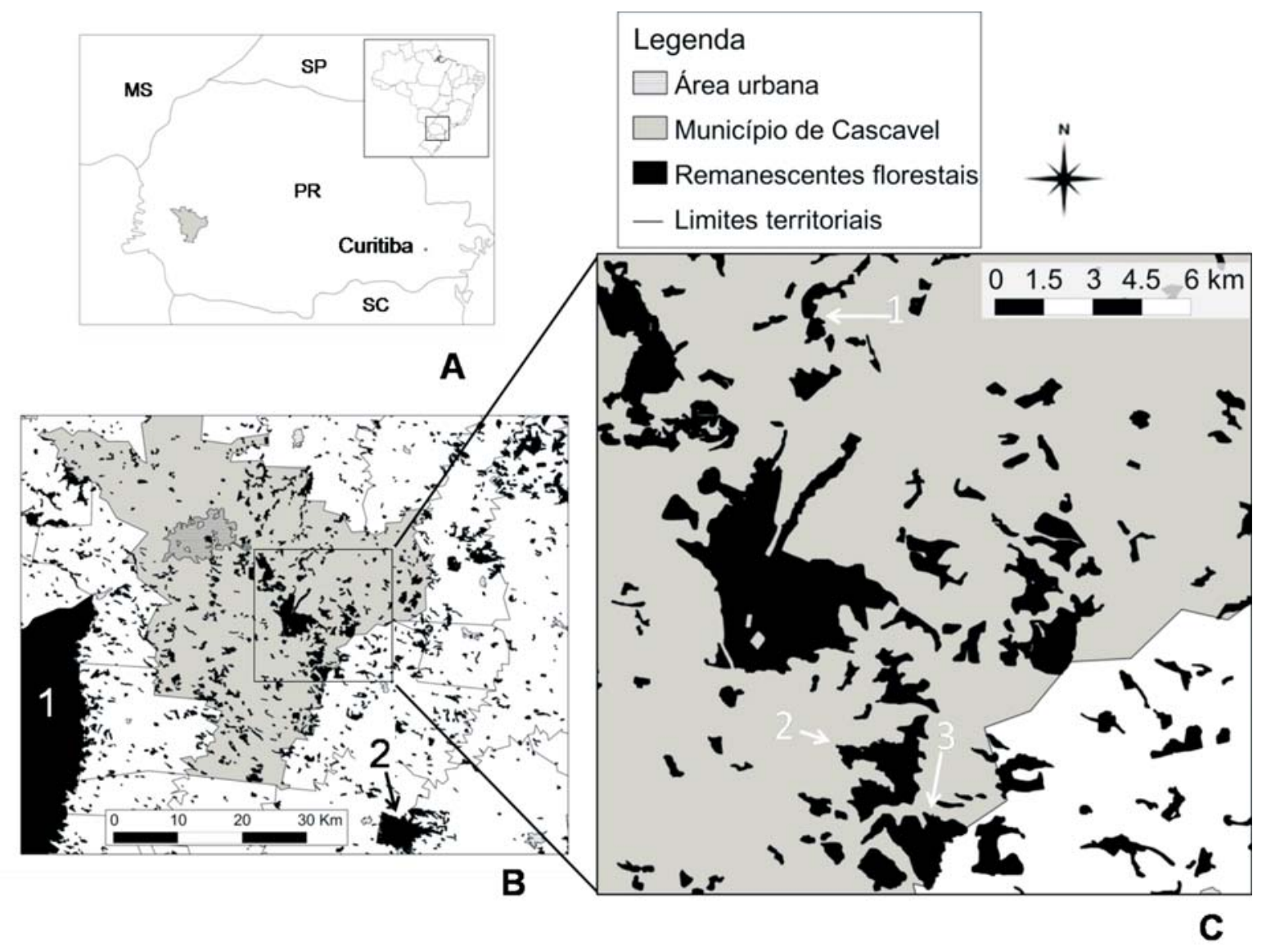

Figura 1 -A) Localização do Município de Cascavel, Paraná, Brasil; B) Remanescentes florestais da região Oeste do Paraná, 1 - Parque Nacional do Iguaçu e 2 - Parque Estadual do Rio Guarani; C) Fragmentos estudados: 1 - CEA, 2 - MRO e 3 - MRT.

Figure 1 - A) Location of Cascavel municipality, Paraná State, Brazil; B) Forest remnants of Western Paraná State, 1 - Iguaçu National Park and 2 - Rio Guarani State Park; C) Fragments studied: 1 - CEA, 2 - MRO and $3-M R T$.

\subsection{Coleta de dados}

Em MRO e MRT, os dados foram coletados de fevereiro a julho de 2007. Em CEA, a coleta de dados foi realizada de janeiro a julho de 2008. Os registros de mamíferos foram realizados de formas direta (visualizações) e indireta (vestígios, análise de pelos e entrevistas com moradores locais).

Para obtenção dos registros das espécies foram inspecionados trilhas, estradas, margens de cursos d’água e acessos no interior e bordas dos fragmentos, em busca de evidências direta e indireta. As pegadas, fezes, tocas e carreiros encontrados foram identificados com base em conhecimento prévio e literatura técnica de referência (BECKER; DALPONTE, 1991; BORGES; TOMÁS, 2004). A utilização de vestígios para registro de médios ( $>1 \mathrm{~kg}$ até $20 \mathrm{~kg}$ ) e grandes mamíferos (> $20 \mathrm{~kg}$ ) é uma técnica eficiente, já que propicia dados em curtos espaços de tempo e necessita de pouca logística e recursos (SILVEIRA et al., 2003).

Fezes de carnívoros também foram coletadas e posteriormente analisadas. Em laboratório, as fezes foram triadas em busca de pelos para identificação de predadores e, ou, das presas (QUADROS, 2002; QUADROS; MONTEIRO-FILHO, 2006). A identificação das espécies se baseou em Quadros (2002) e na coleção de referência do Laboratório de Zoologia de Vertebrados e Biologia da Conservação da Universidade Estadual do Oeste do Paraná. 
Com os dados obtidos em campo e em análises de pelos, foi calculada a frequência de registros das espécies em cada fragmento (exprimida pelo número de registros a cada 10 dias amostrais, Tabela 1).

A fim de complementar os dados obtidos em campo e com análises microscópicas de pelos, foram entrevistados moradores do entorno das áreas amostradas, que residiam até $300 \mathrm{~m}$ dos fragmentos. Durante a entrevista, imagens de espécies com possível ocorrência na região eram apresentadas aos entrevistados para que pudessem reconhecê-las. A utilização de entrevistas é recomendada para complementar levantamento de médios e grandes mamíferos (VOSS; EMMONS, 1996) e vem sendo empregada com sucesso na obtenção de dados (ROCHA et al., 2005; CHEREM, 2005; MICHALSKI, 2010).

Para classificar as espécies citadas nas entrevistas, foi utilizado o Índice de constância de ocorrência (SILVEIRA-NETO et al., 1976), em que as espécies citadas por menos de $25 \%$ dos entrevistados foram consideradas Ocasionais, as citadas por mais de $25 \%$ e menos de $50 \%$ dos entrevistados como Acessórias e as referidas por mais de $50 \%$ foram consideradas Constantes (vide ROCHA-MENDES et al., 2005).

Durante o trabalho de campo foram tomadas notas sobre ameaças locais que incidem sobre as populações de mamíferos, assim como sobre os fragmentos. Também foi analisada a matriz circundante dos fragmentos, através de observação em campo para inferência sobre aspectos relacionados ao grau de isolamento de cada área.

\section{RESULTADOS}

\subsection{Espécies}

No total foram registradas 32 espécies, entre as quais há uma exótica (Lepus europaeus) e duas de pequeno porte (menos de $1 \mathrm{~kg}$ ) (Tabela 1 ), sendo 32 espécies na MRT, 30 na MRO e 24 no CEA (Tabela 1). As espécies registradas estão inseridas em oito ordens e 18 famílias. Nove espécies estão sob o risco de extinção no Estado do Paraná, e cinco configuram com dados insuficientes na Lista de Espécies Ameaçadas do Estado (MARGARIDO; BRAGA, 2004). Três registros puderam ser confirmados apenas para os gêneros Dasypus sp, Leopardus sp e Mazama sp e não foram computados na contagem das espécies (Tabela 1), já que poderiam corresponder àquelas já anotadas.

\subsection{Ameaças e matriz}

Nos fragmentos MRT e MRO foram registradas atividades cinegéticas, como a presença de cães de caça perseguindo animais. Durante parte da realização do trabalho, nesses fragmentos havia "ervateiros" trabalhando na extração da erva-mate (Ilex paraguariensis). Os “ervateiros” possuíam cães, possivelmente utilizados para caçar. Além disso, próximo ao local onde eles pernoitavam foram encontradas penas de aves silvestres. Os moradores do entorno dessas áreas relataram que havia muita caça no local.

Quanto à matriz circundante, os fragmentos MRT e MRO, que distam 400 m entre si, eram delimitados por áreas agrícolas e pecuárias e estavam ligados a outros fragmentos via matas ciliares bem preservadas, porém não havia conexão direta entre essas duas áreas amostradas. Ambos estavam próximos a outros fragmentos grandes de mata, que se encontravam a menos de 1.000 m de distância (Figura 1C).

No CEA não houve registro de caça durante as campanhas amostrais, nem foi mencionada essa atividade nas entrevistas com moradores do entorno. Entretanto, foi constatada a extração de pinhões (sementes de Araucaria angustifolia). Esse fragmento é delimitado em parte por áreas agrícolas e parte por duas rodovias pavimentadas, estando distantes mais de $1.000 \mathrm{~m}$ de grandes fragmentos (Figura 1C). A única conexão direta à outra área florestal era feita via estreita mata ciliar.

\section{DISCUSSÃO}

O número de espécies registradas (32 espécies) corresponde à cerca de $18 \%$ das espécies de mamíferos terrestres com ocorrência confirmada no Estado do Paraná (176 espécies, M. MIRETZKI - comunicação pessoal, em MARGARIDO; BRAGA, 2004). Quando se consideram apenas as 30 espécies terrestres de médio e grande porte, a porcentagem sobe para 58\% (50 espécies nativas e duas exóticas no Paraná, com base em MARGARIDO; BRAGA, 2004; REIS et al., 2006; REIS et al., 2010). Vale salientar que muitas espécies com ocorrência no Estado do Paraná não tinham distribuição natural na região amostrada (MARGARIDO; BRAGA, 2004; REIS et al., 2006; REIS et al., 2010).

Embora estudos mais detalhados sobre o tamanho dessas populações sejam necessários (GALETTI et al., 2009), o grande número de espécies encontradas 
Tabela 1 - Espécies de mamíferos registradas nos fragmentos estudados; e frequência de registros e Índice de constância de ocorrência nas entrevistas ( ${ }^{1}$ espécie exótica, ${ }^{2}$ espécie de pequeno porte, ${ }^{3}$ baseado em Margarido e Braga 2004).

Table 1 - Mammals recorded in the fragments studied; and frequency of records and Occurrence Constancy Index in interviews ( ${ }^{1}$ exotic species, ${ }^{2}$ small mammal species, ${ }^{3}$ based in Margarido and Braga 2004).

\begin{tabular}{|c|c|c|c|c|c|}
\hline \multirow[b]{2}{*}{ Táxon } & \multirow{2}{*}{$\begin{array}{l}\text { Nível de ameaça } \\
\text { regional }^{3}\end{array}$} & \multirow[t]{2}{*}{ Motivo de caça } & \multicolumn{3}{|c|}{ Frequência (Índice de ocorrência) } \\
\hline & & & MRT & MRO & CEA \\
\hline \multicolumn{6}{|l|}{ Artiodactyla } \\
\hline \multicolumn{6}{|l|}{ Cervidae } \\
\hline Mazama americana & DD & Carne & (acess.) & (acess.) & - \\
\hline Mazama gouazoubira & DD & Carne & (ocas.) & (ocas.) & - \\
\hline Mazama nana & $\mathrm{Vu}$ & Carne & (const.) & (const.) & (const.) \\
\hline Mazama sp & - & Carne & 1,5 & 1,0 & 0,5 \\
\hline \multicolumn{6}{|l|}{ Tayassuidae } \\
\hline Pecari tajacu & $\mathrm{Vu}$ & Carne & (ocas.) & (ocas.) & - \\
\hline \multicolumn{6}{|l|}{ Carnivora } \\
\hline \multicolumn{6}{|l|}{ Canidae } \\
\hline Cerdocyon thous & $\mathrm{N}$ & Retaliação/pele & 4,5; (const.) & 5,$5 ;$ (const.) & 1,$5 ;$ (const.) \\
\hline \multicolumn{6}{|l|}{ Felidae } \\
\hline Leopardus pardalis & $\mathrm{Vu}$ & Retaliação/pele & 1,5 (const.) & 1,5 (const.) & 0,5 (const.) \\
\hline Leopardus tigrinus & $\mathrm{Vu}$ & Retaliação/pele & 10,5 (const.) & 7,5 (const.) & (const.) \\
\hline Leopardus $\mathrm{sp}$ & - & Retaliação/pele & 2,0 & 3,0 & 1,5 \\
\hline Puma concolor & $\mathrm{Vu}$ & Retaliação/pele & (ocas.) & (ocas.) & - \\
\hline Puma yagouaroundi & DD & Retaliação/pele & (aces.) & (aces.) & (const.) \\
\hline \multicolumn{6}{|l|}{ Mustelidae } \\
\hline Eira barbara & $\mathrm{N}$ & Retaliação/pele & (const.) & (const.) & (const.) \\
\hline Galictis cuja & $\mathrm{N}$ & Não caçada & (const.) & 0,5 ; (const.) & (const.) \\
\hline Lontra longicaudis & $\mathrm{Vu}$ & Retaliação/pele & (const.) & - & - \\
\hline \multicolumn{6}{|l|}{ Procyonidae } \\
\hline Nasua nasua & $\mathrm{N}$ & Retaliação/pele & 0,5 (const.) & 1,0 (const.) & 1,0 (const.) \\
\hline Procyon cancrivorus & $\mathrm{N}$ & Retaliação/pele & 0,5 (const.) & 1,0 (const.) & 1,5 (const.) \\
\hline \multicolumn{6}{|l|}{ Cingulata } \\
\hline \multicolumn{6}{|l|}{ Dasypodidae } \\
\hline Cabassous tatouay & DD & Carne & (const.) & (const.) & (const.) \\
\hline Dasypus novemcinctus & $\mathrm{N}$ & Carne & (const.) & (const.) & (const.) \\
\hline Dasypus septemcinctus & DD & Carne & (ocas.) & (ocas.) & - \\
\hline Dasypus $\mathrm{sp}$ & - & Carne & 2,0 & 3,0 & 2,5 \\
\hline Euphractus sexcintus & $\mathrm{N}$ & Carne & (const.) & (const.) & (const.) \\
\hline \multicolumn{6}{|l|}{ Didelphimorphia } \\
\hline Dipelphidae & & & & & \\
\hline Didelphis albiventris & $\mathrm{N}$ & Retaliação & (const.) & (const.) & (const.) \\
\hline Didelphis aurita & $\mathrm{N}$ & Retaliação & (const.) & (const.) & (const.) \\
\hline Lagomorpha & & & & & \\
\hline Leporidae & & & & & \\
\hline Lepus europaeus ${ }^{1}$ & $\mathrm{~N}$ & Carne & 0,5 (const.) & 1,5 (const.) & 0,5 (const.) \\
\hline Sylvilagus brasiliensis & $\mathrm{Vu}$ & Carne & (aces.) & (aces.) & - \\
\hline Primates & & & & & \\
\hline Atelidae & & & & & \\
\hline Alouatta guariba clamitans & $\mathrm{Vu}$ & Não caçada & 0,5 (const.) & 0,5 (const.) & - \\
\hline Cebidae & & & & & \\
\hline Cebus nigritus & $\mathrm{N}$ & Não caçada & 1,0 (const.) & 0,5 (const.) & 1,0 (const.) \\
\hline Pilosa & & & & & \\
\hline Myrmecophagidae & & & & & \\
\hline Tamandua tetradactyla & $\mathrm{N}$ & Não caçada & 0,5 (const.) & (const.) & (const.) \\
\hline Rodentia & & & & & \\
\hline Cavidae & & & & & \\
\hline Cavia aperea $^{2}$ & $\mathrm{~N}$ & Não caçada & (const.) & (const.) & 0,$5 ;$ (const.) \\
\hline Hydrochoerus hydrochaeris & $\mathrm{N}$ & Carne & 1,5 ; (const.) & - & - \\
\hline Cuniculidae & & & & & \\
\hline Cuniculus paca & En & Carne & 1,$5 ;$ (const.) & 1,$0 ;$ (const.) & 0,5 ; (const.) \\
\hline Dasyproctidae & & & & & \\
\hline Dasyprocta azarae & $\mathrm{N}$ & Carne & 0,$5 ;$ (const.) & 1,$0 ;$ (const.) & 2,$0 ;$ (const.) \\
\hline Erethizontidae & & & & & \\
\hline Sphiggurus spinosus & $\mathrm{N}$ & Carne & (const.) & (const.) & (const.) \\
\hline Sciuridae & & & & & \\
\hline Guerlinguetus ingrami ${ }^{2}$ & $\mathrm{~N}$ & Não caçada & (const.) & (const.) & 1,$0 ;$ (const.) \\
\hline Myocastoridae & & & & & \\
\hline Myocastor coypus & $\mathrm{N}$ & Não caçada & (const.) & (const.) & 0,$5 ;$ (const.) \\
\hline
\end{tabular}

DD = dados deficientes; $\mathrm{Vu}=$ vulnerável; $\mathrm{En}=$ em perigo; $\mathrm{N}=$ não ameaçada; acess. = acessória; ocas. = occasional; e const. = constante). $D D=$ Deficient data, $\mathrm{Vu}=$ Vulnerable, En = Endangered, $N=$ not threatened; acess. = accessory, ocas. = occasional, const. = constant). 
e o registro de espécies ameaçadas indicam o valor de fragmentos no auxílio à manutenção da biodiversidade, como já vem sendo demonstrado em outros estudos com mastofauna (CHIARELLO, 2000b; ROCHA; DALPONTE, 2006; PRADO et al., 2008). No caso dos fragmentos MRT e MRO, é ressaltado o valor conservacionista da manutenção de áreas de RL e de APP, garantidas pela Lei 4.771/1965, conhecida como Código Florestal, que vem sofrendo pressões políticas para que seja alterada (GALETTI et al., 2010; METZGER et al., 2010). Entretanto, apesar de persistirem nesses ambientes, essas espécies são ameaçadas por ações antrópicas, que são favorecidas pela fragmentação, como é o caso da extração de produtos não madeireiros observada neste estudo: pinhões e erva-mate.

A extração de pinhões para consumo humano é comum na região, sendo prejudicial à conservação da fauna e dos fragmentos, pois limita um recurso muito importante para os animais silvestres em época de escassez alimentar e o recrutamento de Araucaria angustifolia (PALUDO et al., 2009; VIEIRA; IOB, 2009). No caso do CEA, a extração é proibida por se tratar de uma UC de Proteção Integral (Lei nº 9.985/2000). Já a extração de erva-mate em MRO e em MRT é autorizada nas porções correspondentes a RL, sob a forma de manejo florestal (Lei 4.771/1965), mas pode prejudicar o ambiente pela abertura de trilhas e do dossel, aumentando efeitos de borda, como a invasão de lianas e taquaras, impedindo a regeneração florestal (FISZON et al., 2003). Embora, no entanto, a própria ampliação de atividades de silvicultura com espécies arbóreas nativas seja alternativa para aumentar áreas disponíveis para a fauna (FONSECA et al., 2009), desde que técnicas adequadas sejam adotadas para minimizar o impacto ambiental.

A caça, outra atividade favorecida pela fragmentação e constatada neste estudo, tende a causar impactos, sobretudo nas espécies animais de maior porte(CULLEN-JR. et al., 2000; CULLEN-JR. et al., 2001; PERES, 2001). Algumas espécies cinegéticas foram registradas, como é o caso de veados (Mazama sp), cutia (Dasyprocta azarae) e paca (Cuniculus paca), porém outras espécies cuja presença seria esperada na região, como a anta (Tapirus terrestris) e o queixada (Tayassu pecari), não foram registradas. Apesar da grande exigência ecológica, essas espécies podem persistir mesmo em ambientes fragmentados. No Paraná, por exemplo, T. terrestris foi registrada no Parque Estadual da Mata dos Godoy, área de 690 ha, em Londrina, PR (IAP, 2002), e T. pecari em fragmentos e variando de 100 a 500 ha, em São Pedro do Ivaí, PR (G. V. BIANCONI - comunicação pessoal).

Nas entrevistas foi relatado que queixadas viviam nos fragmentos MRO e MRT cerca de 10 anos antes deste estudo, quando eram muito procurados por caçadores, o que pode ter contribuído para a sua extinção local. O queixada tem sido apontado como uma das primeiras espécies a desaparecer sobre forte pressão de caça (PERES, 1996, 2001; CULLEN-JR. et al., 2000), e é provável que outras espécies venham a se extinguir localmente caso a caça não seja combatida. A perda de espécies tem consequências diretas na comunidade vegetal, pois, além de sofrer com os efeitos diretos da fragmentação (TABARELLI et al., 2004; TABARELLI et al., 2010), a vegetação dos fragmentos deve estar sofrendo também mudanças decorrentes do processo de defaunação, pela quebra de interações ecológicas (DIRZO; MIRANDA, 1991; SILMAN et al., 2003; TERBORGH et al., 2008).

Considerando, assim, as implicações que as atividades antrópicas têm sobre os remanescentes florestais, aumento no efetivo de fiscalização é necessário tanto para controlar e monitorar atividades extrativistas quanto para combater a caça ilegal. Ações de educação ambiental, tendo como alvo a população do entorno desses remanescentes, também são importantes, mas requerem tempo maior para resultar em mudança de atitudes.

Fora as pressões antrópicas diretas que os fragmentos sofrem, a própria condição de áreas relativamente isoladas e restritas têm implicações sobre a mastofauna (COSTA et al., 2005). Mais espécies ocorrem nos fragmentos maiores, MRT e MRO, e apenas nessas áreas, espécies essas com porte superior a $20 \mathrm{~kg}$, como cateto (Pecari tajacu), capivara (Hydrochoerus hydrochaeris) e veado-mateiro (Mazama americana). A persistência de espécies em fragmentos é explicada, principalmente, pelo tamanho da área remanescente (CHIARELLO, 1999; MICHALSKI; PERES, 2007), mas fatores como o isolamento também podem estar influenciando nas taxas de recolonização e sobrevivência das espécies (CROOKS, 2002). Como MRT e MRO possuem conectividade maior, possivelmente têm maiores taxas de recolonização, o que acaba refletindo positivamente no número de espécies (GILBERT-NORTON 
et al., 2009). No CEA, a proximidade de rodovias pavimentadas também gera um obstáculo adicional ao deslocamento das espécies, devido ao risco de atropelamentos (CÂNDIDO-JR. et al., 2002; CHEREM et al., 2007).

A ampliação da conectividade desses fragmentos é importante e pode ser feita pela adequação legal de APPs em matas ciliares. Estudos em matas ripárias e corredores vêm demonstrando sua importância no fluxo biológico (PARDINI et al., 2005; GILBERT-NORTON et al., 2009). Como os remanescentes estudados se encontram próximos da área de implantação de um dos ramos do Corredor Iguaçu-Paraná (TOSSULINO et al., 2007), a ampliação desse corredor para interceptar os fragmentos estudados, assim como outros remanescentes florestais da região, seria muito importante não só pelo número de espécies encontradas localmente, mas pela porção de mata nativa que neles seria preservada. Ademais, com a demarcação de um corredor bem planejado e monitorado é possível realizar a conexão das áreas estudadas com o Parque Estadual do Rio Guarani e com o Parque Nacional do Iguaçu, permitindo um fluxo maior de fauna proveniente desses parques (ver Figura 1B).

Outro empecilho à conservação de mamíferos na região Oeste do Paraná é a falta de UCs (MARGARIDO; BRAGA, 2004). Do restante das áreas de ocorrência de FOM (RIBEIRO et al., 2009), somente pequena parcela está protegida sob UCs de Proteção Integral, o que corresponde, hoje, à cerca de $4 \%$ da área original de FOM do Oeste paranaense (C. R. BROCARDO, dados não publicados). A ampliação de áreas legalmente protegidas, juntamente com o manejo de áreas particulares, constitui medida mais eficiente para a conservação de mamíferos e outros grupos de vertebrados, assim como da própria FOM (CHIARELLO, 2000b; INDRUSIAK; MONTEIRO, 2009).

Portanto, a garantia da conservação de mamíferos no contexto onde foi realizado este estudo depende de um conjunto de medidas públicas que vão desde a fiscalização e a criação de programas de educação ambiental até a implantação de corredores de biodiversidade e de novas UCs. Os resultados reforçam a ideia do importante papel dos fragmentos florestais na conservação de mamíferos, sobretudo na Mata dos Pinhais, formação vegetal extremamente ameaçada e ainda pouco conhecida.

\section{AGRADECIMENTOS}

À Agropecuária Festugato Ltda., pela autorização da pesquisa nas reservas de mata localizadas em sua propriedade; gratos a todos os funcionários, pelo auxílio e pela atenção, em especial a Domingos Andreolla (in memoriam); à Secretaria Municipal de Meio Ambiente de Cascavel, pela autorização da pesquisa no Centro de Educação Ambiental Suely Marcondes de Moura Festugato; a F. Rocha-Mendes e G. V. Bianconi, pela revisão do manuscrito e sugestões; aos dois revisores anônimos, pelas sugestões; e ao CNPq, pela concessão de bolsa a C.R.B. durante a redação deste artigo.

\section{REFERÊNCIAS}

BECKER, M.; DALPONTE, J. C. Rastros de mamíferos silvestres brasileiros: um guia de campo. Brasília: Universidade de Brasília, 1991. 181p.

BORGES, P. A. L.; TOMÁS, W. M. Guia de rastros e outros vestígios de mamíferos do Pantanal. Corumbá: Embrapa, 2004. 139p.

CÂNDIDO JR, J. F. The edge effect in a forest bird community in Rio Claro, São Paulo State, Brazil. Ararajuba, v.8, n.1, p.9-16, 2000.

CÂNDIDO JR., J. F. et al. Animais atropelados na rodovia que margeia o Parque Nacional do Iguaçu, Paraná, Brasil, e seu aproveitamento para estudos da Biologia da Conservação. In: CONGRESSO BRASILEIRO DE UNIDADES DE CONSERVAÇÃO, 3., 2002, Fortaleza. Anais... Fortaleza: Rede Pró-Unidades de Conservação Fundação o Boticário de Proteção a Natureza, 2002. v.1. p.553-562.

CAstella, P. R.; BRiteZ, R. M. A floresta com araucária no Paraná: conservação e diagnóstico dos remanescentes florestais. Brasília: Ministério do Meio Ambiente, 2004. 233p.

CHEREM, J. J. Registros de mamíferos não voadores em estudos de avaliação ambiental no sul do Brasil. Biotemas, v.18, n.2, p.169-202, 2005.

CHEREM, J. J. et al. Mamíferos de médio e grande porte atropelados em rodovias do Estado de Santa Catarina, sul do Brasil. Biotemas, v.20, n.3, p.81-96, 2007.

Revista Árvore, Viçosa-MG, v.36, n.2, p.301-310, 2012 
CHIARELLO, A. G. Effects of fragmentation of the Atlantic forest on mammal communities in southeastern Brazil. Biological Conservation, v.89, n.1, p.71-82, 1999.

CHIARELLO, A. G. Density and population size of mammals in remnants of Brazilian Atlantic forest. Conservation Biology, v.14, n.6, p.1649-1657, 2000a.

CHIARELLO, A. G. Conservation value of native forest fragment in region of extensive agriculture. Revista Brasileira de Biologia, v.60, n.2, p.237-247, 2000b.

COSTA, L. P. et al. Mammal conservation in Brazil. Conservation Biology, v.19, n.3, p.672-679, 2005.

CROOKS, K. R. Relative sensitivities of mammalian carnivores to habitat fragmentation. Conservation Biology, v.16, n.2, p.488-502, 2002.

CULLEN JR, L.; BODMER, R. E.; PÁDUA, C. V. Effects of hunting in habitat fragments of the Atlantic Forest, Brazil. Biological

Conservation, v.95, n.1, p.49-56, 2000.

CULLEN JR, L.; BODMER, R. E.; PÁDUA, C. V. Ecological consequences of hunting in Atlantic Forest patches, São Paulo, Brazil. Oryx, v.35, p.137-144, 2001.

DIRZO, R.; MIRANDA, A. Altered patterns of herbivory and diversity in forest understory: a case study of the possible consequences of contemporary defaunation. In: PRICE, P. W. et al. (Eds.). Plant-Animal interactions: evolutionary ecology in tropical and temperate regions. New York: John Wiley \& Sons, 1991. p.273-287.

FAHRIG, L. Effects of habitat fragmentation on biodiversity. Annual Reviews in Ecology, Evolution and Systematics, v.34, p.487515, 2003.

FISZON, J. T.; MARCHIORO, N. P. X.; BRITEZ, R. M. Causas antrópicas. In: RAMBALDI, D. M.; OLIVEIRA, D. A. S. (Eds.). Fragmentação de ecossistemas: causas, efeitos sobre a biodiversidade e recomendações de políticas públicas. Brasília: MMA/SBF, 2003. p.65-99.
FONSECA, C. R. et al. Princípios modernos de manejo florestal e a conservação da biodiversidade associada à Floresta com Araucária. In: FONSECA, C. R. et al. (Eds.). Floresta com Araucária: ecologia, conservação de desenvolvimento sustentável. Ribeirão Preto: Holos, 2009. p.287-295.

GALETTI, M. et al. Priority areas for conservation of Atlantic Forest large mammals. Biological Conservation, v.142, p.1229-1241, 2009.

GALETTI, M. et al. Mudanças no Código Florestal e seu impacto na ecologia e diversidade dos mamíferos no Brasil. Biota Neotropica, v.10, n.4, p.47-52, 2010. Disponível em: <http:// www.biotaneotropica.org.br/v10n4/pt/ fullpaper?bn00710042010+pt $>$ acessado em: 28 de jan. de 2011.

GILBERT-NORTON, L. et al. A meta-analytic review of corridor effectiveness. Conservation Biology, v.24, n.3, p.660-668, 2009.

GREGORY, V.; MYSKIO, A. M.; GREGORY, L. T. M. Porto Britânia a Pato Bragado: memórias e histórias. Marechal Cândido Rondon: Germânica, 2004. 200p.

INSTITUTO AMBIENTAL DO PARANÁ - IAP. Plano de Manejo do Parque Estadual Mata dos Godoy, 2004. Disponível em: $<\underline{\text { http:// }}$ www.uc.pr.gov.br/modules/conteudo/ conteudo.php?conteudo $=27>$ acessado em: 24 de out. de 2010 .

INDRUSIAK, C.; MONTEIRO, S. A. Unidades de Conservação na área de distribuição da Araucária. In: FONSECA, C. R. et al. (Eds.). Floresta com Araucária: ecologia, conservação de desenvolvimento sustentável. Ribeirão Preto: Holos, 2009. p.253-265.

KOCH, Z.; CORRÊA, M. S. Araucária: a floresta do Brasil meridional. Curitiba: Olhar Brasileiro, 2002. 148p.

Lei 4.771 de 15 de setembro de 1965. Disponível em: <http://www.planalto.gov.br/ccivil 03/Leis/

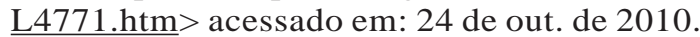

Lei 9.985 de 18 de julho de 2000. Disponível em: <http://www.planalto.gov.br/ccivil_03/Leis/

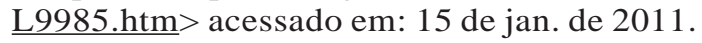


MÄHLER JR, J. K. F.; LAROCCA, J. F. Fitosionomias, desmatamento e fragmentação da Floresta com Araucária. In: FONSECA, C. R. et al. (Eds.). Floresta com Araucária: ecologia, conservação de desenvolvimento sustentável. Ribeirão Preto: Holos, 2009. p.243-252.

MARGARIDO, T. C. C.; BRAGA, F. G. Mamíferos. In: MIKICH, S. B.; BÉRNILS, R. S. (Eds.). Livro vermelho da fauna ameaçada no Estado do Paraná. Curitiba: Instituto Ambiental do Paraná, 2004. p.27-142.

METZGER, J. P. et al. Brazilian law: full speed in reverse? Science, v.329, p.276-277, 2010.

MICHALSKI, F. The bush dog Speothos venaticus and short-eared dog Atelocynus microtis in a fragmented landscape in Southern Amazonia. Oryx, v.44, n.2, p.300-303, 2010.

MICHALSKI, F.; PERES, C. A. Disturbancemediated mammal persistence and abundancearea relationships in Amazonian forest

fragments. Conservation Biology, v.21, n.6, p.1626-1640, 2007.

PALUDO, G. F. et al. Estrutura demográfica e padrão espacial de uma população natural de Araucaria angustifolia (Bertol.) Kuntze (Araucariaceae), na Reserva Genética Florestal de Caçador, Estado de Santa Catarina. Revista Árvore, v.33, n.6, p.1109-1121, 2009.

PARDINI, R. et al. The role of forest structure, fragment size and corridors in maintaining small mammal abundance and diversity in an Atlantic forest landscape. Biological Conservation, v.124, p.253-266, 2005.

PERES, C. A. Population status of whitelipped Tayassu pecari and collared peccaries T. tajacu in hunted and unhunted amazonian forests. Biological Conservation, v. 7 , p.115-123, 1996.

PERES, C. A. Synergistic effects of subsistence hunting and habitat fragmentation on Amazonian Forest vertebrates. Conservation Biology, v.5, n.6, p.1490-1505, 2001.
PRADO, M. R.; ROCHA, E. C.; GIUDICE, G. M. L. Mamíferos de médio e grande porte em um fragmento de Mata Atlântica, Minas Gerais, Brasil. Revista Árvore, v.32, n.4, p.741-749, 2008.

QUADROS, J. Identificação microscópica de pêlos de mamíferos brasileiros e sua aplicação no estudo da dieta de carnívoros. 2002. 127f. Tese (Doutorado em Zoologia) - Universidade Federal do Paraná, Curitiba, 2002.

QUADROS, J.; MONTEIRO-FILHO, E. L. A. Coleta preparação de pêlos de mamíferos para a identificação em microscopia óptica. Revista Brasileira de Zoologia, v.23, n.1, p.274278, 2006.

REIS, N. R. et al. (Eds.). Mamíferos do Brasil. Londrina: Universidade Estadual de Londrina, 2006. 437p.

REIS, N. R. et al. (Eds.). Mamíferos do Brasil: guia de identificação. Rio de Janeiro: Technical Books, 2010. 560p.

RIBEIRO, M. C. et al. The Brazilian Atlantic Forest: how much is left, and how is the remaining forest distributed? Implications for conservation. Biological Conservation, v.142, p.1141-115, 2009.

ROCHA, E. C.; DALPONTE, J. C. Composição e caracterização da fauna de mamíferos de médio e grande porte em uma pequena reserva de Cerrado no Mato Grosso, Brasil. Revista Árvore, v.30, n.4, p.669-678, 2006.

ROCHA-MENDES, F. et al. Mamíferos do município de Fênix, Paraná, Brasil: etnozoologia e conservação. Revista Brasileira de Zoologia, v.22, n.4, p.991-1002, 2005.

SILMAN, M. R.; TERBORGH, J. W.; KILTIE, R. A. Population regulation of a dominant rain forest tree by a major seed predator. Ecology, v.84, n.2, p.431-438, 2003.

SILVEIRA, L.; JÁCOMO, A. T. A.; DINIZFILHO, J. A. F. Camera trap, line transect census and track surveys: a comparative evaluation. Biological Conservation, v.114, p.351-355, 2003.

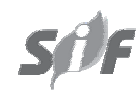

Revista Árvore, Viçosa-MG, v.36, n.2, p.301-310, 2012 
SILVEIRA-NEtO, S. et al. Manual de ecologia dos insetos. São Paulo: Agronômica Ceres, 1976. 419p.

SPERANÇA, A. Cascavel a história. Curitiba: Lagarto, 1992. 321p.

TABARELLI, M. et al. Effects of pioneer tree species hyperabundance on forest fragments in Northeastern Brazil. Conservation Biology, v.24, n.6, p.1654-1663, 2010.

TABARELLI, M. et al. Desafios e oportunidades para a conservação da biodiversidade na Mata Atlântica brasileira. Megadiversidade, v.1, n.1, p.132-138, 2005.

TABARELLI, M.; SILVA, J. M. C.; GASCON, C. Forest fragmentation, synergisms and the impoverishment of neotropical forests.

Biodiversity and Conservation, v.13, p.1419-1425, 2004.

TERBORGH, J. et al. Tree recruitment in an empty forest. Ecology, v.89, n.6, p.1757-1768, 2008.
TOSSUlinO, M. G. P. et al. Resumo executivo da avaliação ecológica rápida do Corredor Iguaçu-Paraná. Curitiba: Instituto Ambiental do Paraná, 2007. 48p. Disponível em: http:// www.prbiodiversidade.pr.gov.br/modules/ biblioteca/uploads/livreto-iguacu.pdf, acessado em: 24 de out. de 2010.

TURNER, I. M. Species loss in fragments of tropical rain forest: a review of the evidence. Journal of Applied Ecology, v.33, p.200-209, 1996.

VIEIRA, E. M.; IOB, G. Dispersão e predação de sementes de Araucaria angustifolia. In: FONSECA, C. R. et al. (Eds.). Floresta com Araucária: ecologia, conservação de desenvolvimento sustentável. Ribeirão Preto: Holos, 2009. p.85-95.

VOSS, R. S.; EMMONS, L. H. Mammalian diversity in Neotropical lowland rainforests: a preliminary assessment. New york: Bulletin of the American Museum of Natural History, 1996. 115p. 